\title{
Controle e Sincronização de Neurônios Acoplados em uma Configuração Linear
}

\author{
Lucas Campanari Simplicio dos Santos* \\ Instituto de Física, USP, \\ 05508-090, São Paulo, SP \\ E-mail: lucas.campanari.santos@usp.br \\ Dr. Elbert E. N. Macau \\ LAC/CTE, INPE \\ 12227-010, São José dos Campos, SP \\ E-mail: elbert@lac.inpe.br

\section{Dr. Epaminondas Rosa, Jr} \\ Physics Department, Illinois State University, USA \\ Normal, IL 61790-4560 \\ E-mail: erosa@ilstu.edu
}

\section{$\underline{\text { RESUMO }}$}

A dinâmica de neurônios acoplados é de interesse em diversas situações práticas reais, desde convulsões e distúrbios do sono até Mal de Alzheimer e depressão [2]. Neurônios em sincronia, por exemplo, seriam desejáveis nos estágios do sono, mas não seriam bem-vindos em casos relacionados a neuropatologias, como o Mal de Parkinson [1].

Nesta etapa do projeto, utilizou-se o modelo de Huber-Braun (HB) para investigar a dinâmica de uma rede de neurônios linearmente acoplados via gap junction que pode, eventualmente, levá-los à sincronia, ou não.

A simulação do modelo de HB [4], que é uma modificação das equações de Hodkin-Huxley [3], é capaz de reproduzir atividade neural em uma vasta gama de configurações de parâmetros [5] fisiológicamente relevantes. Particularmente, observou-se que é possível obter diferentes comportamentos neurais variando-se apenas o parâmetro relativo à repolarização lenta do potencial de ação $\left(g_{\mathrm{sr}}\right)$. Os resultados das simulações numéricas indicam como o acoplamento entre neurônios pode levá-los à um estado síncrono ou não-síncrono, dependendo dos estados dinâmicos de cada neurônio e da força de acoplamento $\left(g_{c}\right)$, como mostra o painel da Figura 1. Foi encontrado que uma constante de acoplamento suficientemente alta faz com que os neurônios que experimentam essa conexão se sincronizem.

Em uma rede de três neurônios, linearmente acoplados, os elementos das extremidades influenciam um ao outro, embora estejam conectados indiretamente por um neurônio central. Analisando o mapa de sincronia desses três neurônios, observou-se que - para determinados valores de constante de acoplamento - pode haver sincronia entre os neurônios das extremidades sem que haja sincronia com o neurônio que os conecta (Figura 2). Foi constatado também que o aumento de uma das constantes de acoplamento, enquanto mantendo a outra fixa, pode implicar na perda de alguns casos de sincronia, o

*bolsista de Iniciação Científica PIBIC/CNPq 
que contradiz a idéia intuitiva de que o aumento da força de acoplamento necessariamente aumenta o número de elementos do estado síncrono.
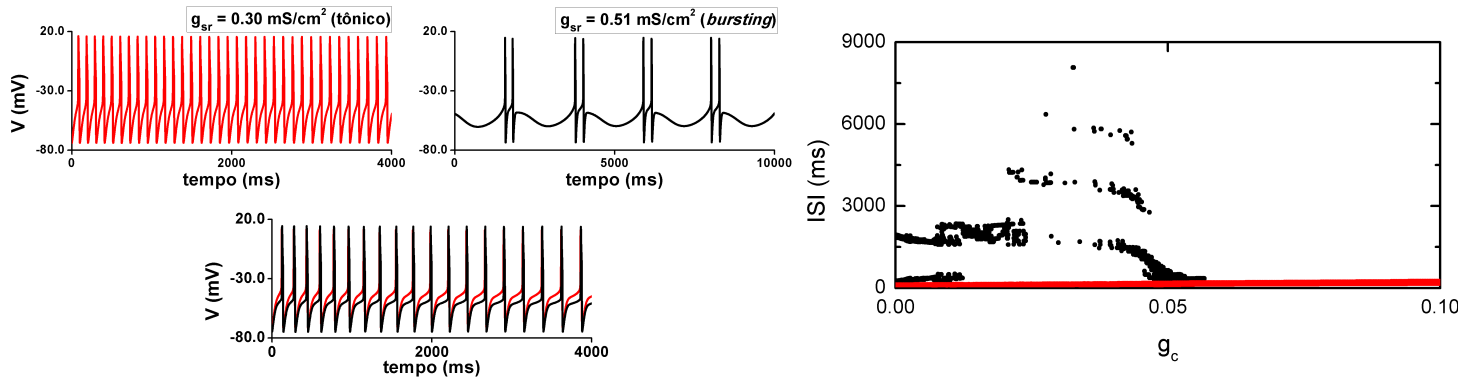

Figura 1: Séries temporais e diagrama de ISI (interspike intervals) com a variação da constante de acoplamento $\left(g_{c}\right)$. As séries temporais superiores mostram o comportamento dos neurônios não-acoplados $\left(g_{c}=0\right)$; a da esquerda refere-se ao estado de atuação neural tônico $\left(g_{\mathrm{sr}}=0.30 \mathrm{mS} / \mathrm{cm}^{2}\right)$ e, a da direita, ao bursting $\left(g_{\mathrm{sr}}=0.51 \mathrm{mS} / \mathrm{cm}^{2}\right)$. A série temporal inferior mostra a dinâmica resultante quando os neurônios estão sincrozados $\left(g_{c}=0.1\right)$. O diagrama de ISI versus $g_{c}$ mostra como os intervalos de tempo entre cada potencial de ação evolui com variação de $g_{c}$.

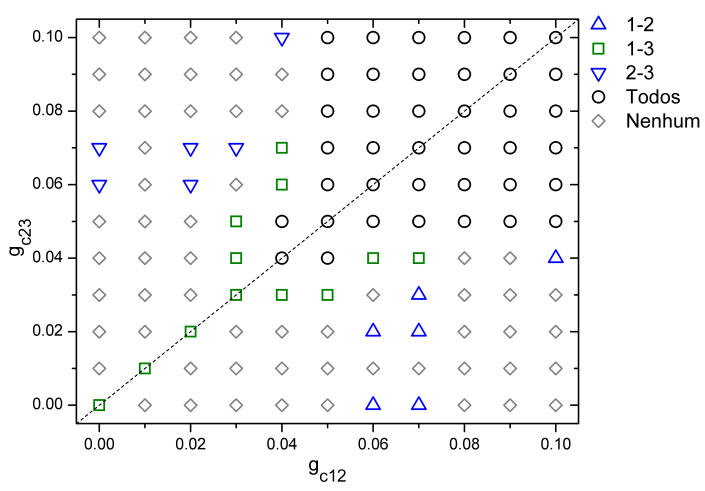

Figura 2: Exemplo de mapa de sincronia de uma rede de três neurônios acoplados bidirecionalmente em uma configuração linear. O neurônio central tem $g_{\mathrm{sr}}=0.51 \mathrm{e}$, os das extremidades, $g_{\mathrm{sr}}=0.30$, ambos $\mathrm{em} \mathrm{mS} / \mathrm{cm}^{2}$. A linha tracejada facilita a vizualização da simetria esperada.

Palavras-chave: Controle, Sincronização, Neurônios Acoplados

\section{Referências}

[1] B.P. Bergstrom, S.G. Sandberg, M. Andersson, J. Mithyantha, F.I. Carrol, and P.A. Garris. Functional reorganization of the presynaptic dopaminergic terminal in parkinsonism. Neuroscience, 193, 2011.

[2] B.A. Heidenreich and T.C. Napier. Effects of serotonergic 5-ht $\mathrm{h}_{1 \mathrm{~A}}$ and 5-ht $\mathrm{h}_{1 \mathrm{~B}}$ ligands on ventral pallidal neuronal activity. NeuroReport, 11, 2000.

[3] A. L. Hodgkin and A. F. Huxley. A quantitative description of membrane current and its application to conduction and excitation in nerve. J. Physiol., 117(4):500-544, 1952.

[4] M. T. Huber, J.C. Krieg, M. Dewald, K. Voigt, and H. A. Braun. Stimulus sensitivity and neuromodulatory properties of noisy intrinsic neuronal oscillators. Biosystems, 48(1-3):95-104, 1998.

[5] S. Postnova, B. WollWeber, K. Voigt, and H. Braun. Impulse pattern in bi-directionally coupled model neurons of different dynamics. Biosystems, 89(1-3):135-142, 2007. 